CWRU-P16-99

\title{
Geometry and Destiny
}

\author{
Lawrence M. Krauss ${ }^{1}$ and Michael S. Turner ${ }^{2,3}$ \\ ${ }^{1}$ Departments of Physics and Astronomy \\ Case Western Reserve University \\ Cleveland, $\mathrm{OH}$ 44106-7079 \\ e-mail:krauss@theory1.phys.cwru.edu \\ ${ }^{2}$ Departments of Astronomy 83 Astrophysics and of Physics \\ Enrico Fermi Institute, The University of Chicago, Chicago, IL 60637-1433 \\ e-mail: mturner@oddjob.uchicago.edu \\ ${ }^{3}$ NASA/Fermilab Astrophysics Center \\ Fermi National Accelerator Laboratory, Batavia, IL 60510-0500 \\ (written for the Gravity Research Foundation Essay Competition)
}

\begin{abstract}
SUMMARY
The recognition that the cosmological constant may be non-zero forces us to reevaluate standard notions about the connection between geometry and the fate of our Universe. An open Universe can recollapse, and a closed Universe can expand forever. As a corollary, we point out that there is no set of cosmological observations we can perform that will unambiguously allow us to determine what the ultimate destiny of the Universe will be.
\end{abstract}


The traditional philosophy of General Relativity is that Geometry is Destiny. We teach undergraduates that the Universe can exist in one of three different geometries, open, closed and flat, and that once we determine which describes our Universe, this fixes its fate.

In the past few years, however, several features of conventional wisdom in cosmology have fallen by the wayside. By 1995 it was already clear that fundamental observables, from the age of the Universe, to the baryon content, and the nature of large-scale structure, all independently pointed to the possible existence of a nonzero cosmological constant [1]. At the very least, there is now definitive evidence that matter, be it dark or luminous, is not sufficiently abundant to result in a flat Universe today [2]. If we are to believe one of the generic predictions of inflation - that we live in an almost exactly flat Universe - a cosmological constant, or some form of energy very much like it is the only way out.

This speculation received dramatic support a year ago, with independent claims by two groups that Type 1a supernova, when used as standard candles, indicated that the expansion of the Universe is accelerating [3, 4]. The simplest explanation of this result is the presence of a cosmological constant.

Most recently, observations of the Doppler peak in the Cosmic Microwave Background anisotropies have begun to provide more definitive evidence that we live in a flat Universe today [5]. When this fact is combined with the SN 1a data, and the data from large-scale clustering, a parameter range of $\Omega_{M} \approx 0.3-0.4$ and $\Omega_{\Lambda} \approx 0.6-0.7$ appears to be strongly favored [6].

While it is premature to claim, on the basis of the existing data, that a $\Lambda$ dominated flat model actually describes our Universe, it is not premature to explore its possible ramifications. Recently, for example, an analysis has been performed that suggests that this observation will have important implications for the future of life in our Universe [7]. Here we focus on a more general feature associated with the incorporation of a cosmological constant into our models: The one-to-one correspondence between geometry and evolution is forever lost.

The mathematical basis of this is described simply. Einstein's equations imply, 
for an isotropic and homogeneous Universe, the following evolution equations for the cosmic scale factor, $R(t)$ :

$$
\begin{aligned}
H^{2} \equiv\left(\frac{\dot{R}}{R}\right)^{2} & =\frac{8 \pi G}{3} \rho_{\text {Тот }}-\frac{k}{R^{2}} \\
\frac{\ddot{R}}{R} & =-\frac{4 \pi G}{3} \sum_{i} \rho_{i}\left(1+3 w_{i}\right)
\end{aligned}
$$

where $k$ is the signature of the 3-curvature, the pressure in component $i$ is related to the energy density by $p_{i}=w_{i} \rho_{i}$ and the total energy density $\rho_{\mathrm{TOT}}=\sum_{i} \rho_{i}$. The evolution of the energy density in component $i$ is determined by

$$
\frac{d \rho_{i}}{\rho_{i}}=-3\left(1+w_{i}\right) \frac{d R}{R} \Rightarrow \rho_{i} \propto R^{-3\left(1+w_{i}\right)}
$$

All forms of normal matter satisfy the strong-energy condition, $\left(\rho_{i}+3 p_{i}\right)=\rho_{i}(1+$ $\left.3 w_{i}\right)>0$, and so if the Universe is comprised of normal matter, the expansion of the Universe always decelerates, cf. Eq. (2). Also, since $\rho$ is positive for normal matter, the first equation implies that $\dot{R} / R$ remains positive and non-zero if $k \leq 0$, and thus the Universe expands forever. Equation (3) and the strong-energy condition imply that $\rho_{i}$ decreases more rapidly than $R^{-2}$. Thus, for $k>0$ there is necessarily a turning point with $H=0$ and $\ddot{R}<0$, and the Universe must ultimately recollapse. Geometry determines destiny.

However, a cosmological constant violates the strong-energy condition, completely obviating the logic of the above argument. Recalling that $p_{\Lambda}=-\rho_{\Lambda}$ for a cosmological term, and that $p_{M}=0$ for matter, the above equations become,

$$
\begin{aligned}
H^{2} & =\frac{8 \pi G}{3}\left(\rho_{M}+\rho_{\Lambda}\right)-\frac{k}{R^{2}} \\
\frac{\ddot{R}}{R} & =-\frac{4 \pi G}{3}\left(\rho_{M}-2 \rho_{\Lambda}\right)
\end{aligned}
$$

Since $\rho_{\Lambda}=$ constant, while $\rho_{M} \propto R^{-3}$, even if $k>0$, as long as $H>0$ when $\rho_{\Lambda}$ comes to dominate the expansion, it will remain positive forever, and as is well known, the expansion will ultimately accelerate, $R(t) \rightarrow e^{H t}$ with $H=\sqrt{8 \pi G \rho_{\Lambda} / 3}$. 
One conventionally defines the scaled energy density $\Omega \equiv \rho_{\text {Tот }} / \rho_{\text {crit }}=8 \pi G \rho / 3 H^{2}$, so that $\Omega-1=k / H^{2} R^{2}$. Thus the sign of $k$ is determined by whether $\Omega$ is greater than or less than 1 . In this way, a measurement of $\Omega$ at any epoch - including the present - determines the geometry of the Universe. However, we can no longer claim that the magnitude of $\Omega$ uniquely determines the fate of the Universe.

This decoupling between $\Omega$ and destiny can also be seen using Sandage's deceleration parameter $q \equiv-(\ddot{R} / R) / H^{2}$, which, by using Eqs. (1).2), can be written as

$$
q=\frac{\Omega}{2}+\frac{3}{2} \sum_{i} w_{i} \Omega_{i}
$$

The sign of $q$, and thus the deceleration of the Universe at any given epoch depends upon the equation of state and not on $\Omega$ alone.

While in the presence of a cosmological constant, $\Omega$ no longer determines the ultimate fate of the Universe, it is useful in determining how small a cosmological constant could be at the present time and still stop the eventual collapse of a closed Universe. For a closed, matter-only Universe, the scale factor at turnaround is

$$
R / R_{0}=\frac{\Omega_{0}}{\Omega_{0}-1}
$$

While all the evidence today suggests that $\Omega_{0} \leq 1$, existing uncertainties could allow $\Omega_{0}$ to be as large say as 1.1. For $\Omega_{0}=1.1$ the scale factor at turnaround is $11 R_{0}$. Since the density of matter decreases as $R^{-3}$, this means that an energy density in a cosmological term as small as $1 / 1000 t h$ the present matter density will come to dominate the expansion before turnaround and prevent forever recollapse. A cosmological constant this small, corresponding to $\Omega_{\Lambda} \sim 0.001$, is completely undetectable by present, or foreseeable observational probes.

Alternatively, it may seem that if we can unambiguously determine that $k<0$ then we are assured the Universe will expand forever. However, this is the case only as long as the cosmological constant is positive. Since we have no theory for a cosmological constant [8], there is no reason to suppose that this must be the case. When the cosmological constant is negative, the energy density associated with the 
vacuum is constant and negative. In this case, from Eqs. (4,5), one can see that not only is the ultimate expansion guaranteed to decelerate, but recollapse is also inevitable, no matter how small the absolute value of $\Omega_{\Lambda}$ is.

Finally, what if we indeed ultimately verify a non-zero cosmological constant at the present time, as current observations suggest? Are we not then guaranteed an eternal expansion? The answer is again no. As is well known, we have no guarantees that what we observe to behave as a cosmological constant is in fact the actual groundstate vacuum-energy density of the Universe. Any scalar field which is not at the minimum of its potential will, as long as the age of the Universe is small compared to the characteristic time it takes for the field to evolve in its potential, mimic a cosmological term in Einstein's equations. Until the field evolves to its ultimate minimum, we cannot derive the asymptotic solution of these equations in order to determine our destiny.

We thus arrive at the following set of possibilities. As this classification makes clear, it is the ultimate equation of state, not geometry, that determines the fate of the Universe. The key consideration is the value of $\ddot{R}$ at any potential turning point (i.e., where $H=0)$. If it is negative, which requires $\sum_{i} \rho_{i}\left(1+3 w_{i}\right)>0$, recollapse occurs; otherwise expansion resumes and continues eternally.

\section{RECOLLAPSE}

1. Closed $(k>0)$ Universe: $\rho_{M}>2 \rho_{\Lambda}$ when $H=0$

2. Open,flat $(k \leq 0)$, or closed $(k>0)$ Universe: $\rho_{\Lambda}<0$

\section{ETERNAL EXPANSION}

1. Closed $(k>0)$ Universe : $\rho_{M}<2 \rho_{\Lambda}$ before $H=0$

2. Open or flat $(k \leq 0)$ Universe: $\rho_{\Lambda} \geq 0$

For the simplest possibility, a Universe with matter and positive cosmological constant, the dividing point between expansion forever and recollapse can be expressed 
simply: Eternal expansion is inevitable if and only if [9]

$$
\Omega_{\Lambda}>4 \Omega_{M}\left\{\cos \left[\frac{1}{3} \cos ^{-1}\left(\Omega_{M}^{-1}-1\right)+\frac{4 \pi}{3}\right]\right\}^{3} .
$$

Given Einstein's association with the cosmological constant, we would be quite remiss in not mentioning the intermediate case, his static Universe. A static, but unstable, cosmological solution obtains for

$$
\rho_{M}=2 \rho_{\Lambda} \quad \text { and } \quad R=k^{1 / 2} / \sqrt{8 \pi G \rho_{\Lambda}}
$$

The above classification can of course be generalized to any other form of energy that violates the strong-energy condition and/or mimics a cosmological constant. In such cases, the equation of state will generally vary with time.

Indeed, because the equation of state of the Universe can change, we may never be confident that any presently inferred dynamical evolution can be extrapolated indefinitely into the future. Put another way: even if the presently inferred cosmological constant turns out to be a red herring, we cannot definitively argue that a closed Universe will recollapse or that an open Universe will expand forever. A smaller, presently unobservable value of $\Lambda$ could always alter the ultimate fate of the Universe. In a true sense therefore, perhaps only knowledge of a fundamental theory of everything, one that predetermines both the asymptotic values of both $\Omega$ and $\Lambda$, will allow us ultimate knowledge of the ultimate state of the Universe. If instead, the fundamental parameters in our observed Universe arise from a probability distribution based on some underlying theory, then the future is ultimately unknowable.

While these features of the Universe have been implicit in Einstein's equations since they were first written down, they tended to disappear from the popular lore shortly after the cosmological constant did. It is therefore important, now that we appear to be living in a Universe with non-zero cosmological constant, to re-acquaint ourselves with their implications.

Before concluding, we note one additional complication brought on by possible quantum field theoretic phenomena. While the 4-geometry of the observable Universe is classically invariant, its 3-geometry depends upon the choice of the constant-time 
hypersurfaces. It is distinctly possible that a change in equation-of-state will also change the natural choice of hypersurfaces, and thus alter the inferred 3-geometry. A well known example involves the nucleation of a bubble of true vacuum in the midst of a background of false vacuum [10]. Observers inside this bubble will infer an open geometry, while those outside the bubble will observe the bubble to collapse into a black hole. In the different cases an infinite spatial region is interchanged with an infinite temporal one. Further, destiny could also change, as the bubble could have nucleated within a Universe destined for recollapse. Of course, under the conditions of the change in the equation of state defined by the situation described above, the future will be truly unknowable for another reason. It is unlikely that any life-form that evolved in one vacuum would survive the transition into the other.

Returning to our central thrust, the result that geometry and destiny are decoupled is in one sense disappointing. The hope that we could, via a finite set of cosmological observations that might be completed within the next decade, determine eternity was very satisfying. Nevertheless, what we lose in predictive power we gain in fundamental excitement. The microphysics that might generate a non-zero cosmological constant or a scalar field that mimics one will no doubt be central to much of the forefront theoretical and experimental research in the next century, if not the next millennium. The new uncertainty in our ultimate destiny thus opens an exciting door that may lead to a deeper understanding of our ultimate origin.

\section{Acknowledgments}

This work was supported in part by the DOE (at Chicago, Fermilab and Case Western Reserve) and by the NASA (at Fermilab through grant NAG 5-7092).

\section{References}

[1] L.M. Krauss and M.S. Turner, Gen. Rel. Grav. 27, 1135 (1995); J.P. Ostriker and P.J. Steinhardt, Nature 377, 600 (1995). 
[2] L.M. Krauss "The New Cosmology and Dark Matter", to appear in Proceedings of Sheffield International Workshop on Dark Matter, Sept 1998 (hep-ph/9807376); N.A. Bahcall and X. Fan, Proc. Nat. Acad. Sci. 95, 5956 (1998); M.S. Turner, Physica Scripta, in press (1999) (astro-ph/9901109).

[3] S. Perlmutter et al, Astrophys. J., in press (1999) (astro-ph/9812133).

[4] A.G. Riess et al, Astron. J. 114, 722 (1998).

[5] See e.g., K. Coble et al, Astrophys. J., submitted (1999) (astro-ph/9902195); C. Lineweaver, Astrophys. J. 505, 69 (1998).

[6] S. Perlmutter, M.S. Turner, and M. White, Phys. Rev. Lett., submitted (1999) (astro-ph/9901052); L.M. Krauss, Astrophys. J. 501, 461 (1998).

[7] L.M. Krauss and G.D. Starkman, Astrophys. J., submitted (1999) (astroph/9902195).

[8] S. Weinberg, Rev. Mod. Phys. 61, 1 (1989).

[9] S.M. Carroll, W.H. Press and E.L. Turner, Ann. Rev. Astron. E6 Astrophys. 30, 499 (1992).

[10] A. Guth and E.J. Weinberg, Nucl. Phys. B 212, 321 (1983). 